sein - dennoch sollte kein arbeitsrechtlich strukturiertes Gesetz vom Vorrang des Tarifvertrages absehen. Die hier vorgeschlagene Verhandlungspflicht für Tarifverträge wird wohl kaum ernsthaft Bedeutung erlangen. Die Rechtsvergleichung zeigt uns, daß Verhandlungspflichten kein scharfes Instrument sind; auch mögen die rechtspolitischen Folgewirkungen für das Tarifvertragssystem dazu Veranlassung geben, hier erhebliche Bedenken anzumelden.

Es trifft zu, daß ein sehr großer Teil der Frauen in Betrieben und Unternehmen arbeitet, die keine Betriebsräte haben. Genau hier aber wird die Wirkung des Gesetzes am schwächsten sein. Das würde aber durch einen anderen Ansatz und stärker materiellrechtlich formulierte Normen (einschließlich der Kriminalisierung mangelhafter Gleichstellung durch Ordnungswidrigkeitensanktionen) keineswegs besser aussehen. Es ist illusionär zu meinen, die allgemeine Durchsetzungsschwäche geltenden Arbeitsrechts in Betrieben ohne Betriebsrat sei nun ausgerechnet und allein im Bereich der Gleichstellung aufzuheben.

Für die in diesem Gesetzesvorschlag angeführten Sanktionen ist deshalb die Verbandsklage von ganz entscheidender Bedeutung. Gäbe es sie, wären bereits heute geltende Benachteiligungsverbote wegen des Geschlechts erheblich praxiswirksamer. Selbstverständlich gibt ein Verbandsklagerecht den Gewerkschaften nicht nur Rechte, sondern setzt sie auch Anforderungen aus. Allerdings kann und soll es Individualansprüche nicht ersetzen. Es ist deshalb auch nicht damit zu rechnen, daß die Gewerkschaften nun massenhaft als Klägerinnen in Anspruch genommen werden. Aber sie erhalten die Möglichkeit, sich entsprechende Fallgestaltungen herauszusuchen und Klagen anzudrohen - das wird oft genügen - und gegebenenfalls durchzuführen. Sie können damit eine Strategie der Rechtsdurchsetzung zugunsten benachteiligter Frauen entwickeln und diese zum Bestandreil ihres Verbandsinteresses machen.

Im Gesetzesvorschlag nicht enthalten ist eine Gleichstellungsbehörde/beauftragte auf Bundesebene, die - wie die US-amerikanische Equal Employment Opportunity Commission - ein eigenes Klagerecht haben, als Informationszentrale und Netzwerkorganisatorin den Interessenvertretungen der Frauen dienen und dem Bundesdatenschutzbeauftragten vergleichbare Rechte haben könnte. Die Effektivität einer solchen Einrichtung ist zwar sehr von politischen Konjunkturen abhängig, aber solange durchaus zu unterstützen, wie sie nicht anstelle der hier aufgestellten, erheblich effektiveren Regelungen tritt.

\title{
Sibylle Raasch
}

\section{Verhandeltes Recht statt materiellem Gleichstellungsgesetz für die Privatwirtschaft?}

\author{
Anmerkungen zu Heide Pfarr
}

Die Koalitionsvereinbarung zwischen SPD und Bündnis 9o/DIE GRÜNEN vom 20. Oktober 1998 kündigte im Rahmen eines künftigen Aktionsprogramms »Frau und Beruf " noch ein "effektives Gleichstellungsgesetz" mit "verbindlichen Regelungen zur Frauenförderung... auch in der Privatwirtschaft « an.' Im tatsächlich 
vorgelegten Programm »Frau und Beruf « vom Juni 1999 ist dann allerdings nur noch davon die Rede, für den Bereich der Bundesverwaltung solle das »Gleichberechtigungsgesetz von 1994 novelliert und effektiver gestaltet« werden. Für den Bereich der Privatwirtschaft gälte es hingegen, "einen Veränderungsprozess in der Wirtschaft in Richtung auf vereinbarte Zielvorstellungen zu organisieren und dafür den gesetzlichen Handlungsrahmen bereitzustellen. Im Dialog mit der Wirtschaft und den Gewerkschaften sollen nunmehr gleichstellungspolitische Regelungen erarbeitet werden, »die geeignet sind, die tatsächliche Gleichberechtigung herzustellen und zugleich der Unterschiedlichkeit der Unternehmen Rechnung zu tragen«. Bei aller Vielfalt im Vorgehen, ob z. B. durch Tarifverträge oder Betriebsvereinbarungen, sollten allerdings zumindest bestimmte Regelungsziele zugunsten von Frauen eingehalten werden: Beseitigung bestehender Diskriminierung, Berufsausbildung in zukunftssicheren Berufen; verbesserte Arbeitsbedingungen, Erhöhung des Beschäftigungsanteils in Bereichen mit Frauenunterrepräsentanz, Lohngerechtigkeit, Interessenvertretung von Frauen bezogen auf das Gleichstellungsziel. ${ }^{2}$

Die in der KJ dokumentierten Eckpunkte von Heide Pfarr sind als ein erster Konkretisierungsvorschlag aus dem gewerkschaftlichen Bereich ${ }^{3}$ (allerdings nicht $» d e r$ Gewerkschaften «) in diese neue Richtung zu verstehen. Der Vorschlag markiert den Beginn, keinesfalls aber das Ende einer neuen Diskussion um »verhandeltes Recht « im Gleichstellungsbereich, wie erste kritische Reaktionen aus dem frauenpolitisch interessierten Umfeld zeigen. ${ }^{4}$

Bündnis 90/DIE GRÜNEN, der »Juniorpartner« der derzeitigen Regierungskoalition, der sich angesichts negativer Wahltrends dringend in angestammten Politikfeldern wie Gleichstellungspolitik und Umwelt mit eigenen Vorschlägen profilieren müsste, hat in der Vergangenheit wiederholt Entwürfe und Vorüberlegungen für umfassende Antidiskriminierungs- und Gleichstellungsgesetze entwickelt ${ }^{5}$, die in eine ganz andere Richtung wiesen: Neben einem verbesserten Diskriminierungsschutz wurde hier auf Gleichstellungspolitik über sanktionierte Frauenquoten, Gleichstellungspläne und Gleichstellungsbeauftragte mit fest umrissenen Aufgaben und Rechten gesetzt. In dieselbe Richtung wies auch noch ein Entwurf der SPDBundestagsfraktion aus der vergangenen Legislaturperiode. ${ }^{6}$

Ist der im neuen Regierungsprogramm erkennbare und im Eckpunkte-Papier erstmals konkretisierte radikale Paradigmenwechsel in der Gleichstellungspolitik weg von einer materiell regelnden Gleichstellungsgesetzgebung hin zu einem »verhandelten Recht «, für welches das Gesetz nur noch prozedurale Vorgaben macht, lediglich

Sozialdemokratischen Partei Deutschlands und Bundnıs 9o/DIE GRUNEN, Bonn, 20. Oktober 1998, Text hrsg. von Bundnis 90/DIE GRUNEN, Okt. I 998, S. 43.

2 Bundesministerıum fur Famılie, Senıoren, Frauen und Jugend (Hg.): Die Bundesregierung: Programm "Frau und Beruf «. Aufbruch in der Glerchstellungspolitık, Juni 1999, S. 9, 14 f.

3 Heide Pfarr ist nicht nur Professorin an der Universitat Hamburg, sondern auch in der Geschaftsfuhrung der Hans-Bockler-Stiftung und Wissenschaftliche Direktorin des dortigen Wirtschafts- und Sozialwissenschaftlichen Instituts.

4 Vgl. Degen, Barbara: Verwirrende Botschaften: Das geplante Gleıchberechtigungsgesetz fur die Privatwirtschaft, in: Heinrich Boll Stiftung ( $\mathrm{Hg}$.): Erfolgreich in die Chefetagen - Anforderungen an ein Gleichberechtıgungsgesetz fur dıe Privatwirtschaft. Materialsammlung zu der ExpertInnentagung am 2. 7. I 999, S. 22 ff.; Ploger, Elke: Fravenforderung in Unternehmen - frelwillig oder gesetzlich sanktioniert? Die Rechte und Aufgaben der Frauenbeauftragten, in: Heinrich Boll Stiftung a.a.O., S. $34 \mathrm{ff}$.

s Siehe z. B. Die Grunen ( $\mathrm{Hg}$.): Vorlaufıger Entwurf eınes Antidıskrıminierungsgesetzes, 2. Aufl. Bonn i 986 ; Entwurf eines Gesetzes zur Aufhebung der Benachteiligung von Frauen in allen gesellschaftlıchen Bereichen, insbesondere in der Erwerbsarbeit (Antidıskriminierungsgesetz Teil I-ADG I), BTDrs. I I/3266 vom 7. x1. 1988; Peter, Gaby: Entwrurf exnes Gesetzes zur Aufhebung der Benachteiligung von Frauen um Erwerbsleben (Arbeitsrechtlıches Anti-Diskrıminierungsgesetz), Entwurf i 993 erstellt im Auftrag der Bundestagsfraktion Bundnis 90/DIE GRUNEN (unveroffentlichtes Manuskript).

6 Vgl. Entwurf eines Gesetzes zur Gleichstellung von Mann und Frau (Gleichstellungsgesetz), BTDrs. 12/ 5717 vom 22.9. 1993 . 
der Tatsache geschuldet, dass damit Konflikten mit der Privatwirtschaft ausgewichen werden kann? Oder schlägt sich hier eine bessere Einsicht in das aktuell Mach- und Wünschbare nieder? Bei einer Antwort können die bisherigen Erfahrungen mit den bestehenden Gleichstellungsgesetzen für den öffentlichen Dienst einerseits und mit "verhandeltem Recht« in anderen Bereichen andererseits nicht außer Betracht bleiben.

\section{Erfahrungen mit Gleichstellungsgesetzen für den öffentlichen Dienst}

Ende der 8oer Jahre begannen fast alle Bundesländer und 1994 schließlich auch der Bund, für den Bereich ihres öffentlichen Dienstes Gleichberechtigungsgesetze, Gleichstellungsgesetze bzw. Frauenfördergesetze einzuführen.7 Diese Gesetze setzten, allerdings mit unterschiedlichem Verbindlichkeitsgrad, anfangs vor allem darauf, die einzelnen Personalentscheidungen zugunsten von Frauen zu beeinflussen. Im Mittelpunkt - auch der anschließenden juristischen Auseinandersetzungen - stand die Entscheidungsquote unter Qualifikationsvorbehalt, welche in jedem konkreten Einzelfall einer Stellenvergabe die Auswahl zwischen Männern und Frauen steuern sollte. Hinzu kam eine Kontrolle durch Frauenbeauftragte und - später - die Entwicklung von Gleichstellungs- bzw. Frauenförderplänen mit Zielvorgaben. Sanktionen zur Einhaltung von Frauenquoten und Frauenförderplänen blieben allerdings die große Ausnahme. ${ }^{3}$ Angestoßen über $\$_{3}$ Hochschulrahmengesetz und die Landeshochschulgesetze wurde diese Art der Gleichstellungspolitik auch in die Hochschulen implantiert.?

Da diese Gesetze sämtlich Berichtspflichten vorsehen, liegt inzwischen eine Unzahl von Einzelberichten vor, die jedoch jeweils ihren eigenen Berichtskriterien und systematiken folgen und deshalb nur schwer vergleichbar sind. Dem entsprechend fehlt es bisher leider mit Ausnahme des Hessischen und des Niedersächsischen Gleichberechtigungsgesetzes auch an einer umfassenden Auswertung. Gesetzesauswertung sowie Gleichstellungsberichte aus dem öffentlichen Dienst und den Hochschulen konstatieren jedoch Ähnliches: Die gleichzeitig einsetzende vehemente Sparpolitik konterkariere möglicherweise vorhandene Erfolge in Sachen Gleichstellung. Dennoch steige der Frauenanteil im öffentlichen Dienst. Deutliche Erfolge seien jedoch vor allem in der Berufsausbildung und den Einstiegsbereichen bzw. beim wissenschaftlichen Nachwuchs erkennbar und quantitativ belegbar. In den Spitzenpositionen, also im höheren Dienst oder bei Professorenstellen, seien wenig oder gar keine quantitativen Erfolge vorzuweisen. ${ }^{\circ}$ Hinter vorgehaltener Hand wird häufig mit gewisser Verbitterung konstatiert, dass in der Praxis erhebliche Energie der Entscheidungsträger darauf verwendet werde nachzuweisen, warum die vorgegebene

7 Für den Zeitraum bis Ende 1994 immer noch sehr hilfreich die Synopse: Gleichberechrigungsgesetze der Lander und des Bundes, in: STREIT I/Ig9s S. 2 I ff.

8 Ausnahme insbesondere $\$$ ro Abs. 4 und 5 Hessisches Gleschberechrigungsgesetz in der Fassung vom 21. Dez. 1993 (Hess. GVBI I S. 729 ); Ziff. III.2.2.5. Frauenforderrichtlinie der Universitàt Hamburg i.d. Fass. v. 30. Okt. 1997.

9 Z. B. $\ 3$ Abs. 2 Hamburgisches Hochschulgesetz.

Io Vgl. Bednarz-Braun, Iris/Bruhns, Kırsten: Personalpolitsk und Frauenförderung im Offentlichen Dienst, Múnchen 1997; Schon, Christine: Szenarien betrieblicher Gleichstellungspolitik, Konigstein/Taunus I999; Oppen, Maria/Wiechmann, Elke: Frauenförderungsplane unter Reformdruck, WZB Berlin I 998 sowie z. B. Universitat Hamburg, Arbeitsstelle Frauenforderung ( $\mathrm{Hg}$.): Frauenforderung an der Universität Hamburg, 6. Bericht, 1995 , Bericht des Prasidenten S. 6 ff. sowie Bericht der Frauenbeauftragten S. 3 ff., I I ff.; Ministerium fur die Gleichstellung von Frau und Mann des Landes Nordrhein-Westfalen ( $\mathrm{Hg}$.): Dritter und vierter Bericht zum Frauenforderungskonzept, 1993, S. 10 ff., $88 \mathrm{ff}$. 
Frauenförderung nicht funktionieren könne und insbesondere die Quote nicht greife, anstatt sich innovative Gedanken darüber zu machen, wo und wie Gleichstellungspolitik trotz alledem funktionieren könne. Jedenfalls ist dieser Weg zur Gleichstellung mit Widerstand gepflastert und voller zeitaufwendiger bürokratischer Prozeduren.

Neuere Berichte und Pressemeldungen klingen allerdings in der Erfolgsbilanz positiver und konstatieren inzwischen auch in den Spitzenpositionen deutliche Steigerungsraten, bei allerdings weiterhin geringer absoluter Anzahl von Frauen. " Diese Erfolge traten übrigens ein, ohne dass die Gleichstellungsgesetze über effektive Sanktionen verfügten und obwohl Frauen die Einhaltung der Quotierung kaum einklagen konnten ${ }^{12}$, dafür aber Männer zahllose Prozesse gegen die Quoten anstrengten. ${ }^{13}$ Gleichstellungspolitik bedarf offenbar langer Anlaufzeiten und vor allem bewusstseinsbildender Diskurse, bevor sich etwas bewegt. Trotz jüngster Erfolge wird in neueren Gleichstellungsberichten dennoch dafür plädiert, Gleichstellungspolitik müsse sich im öffentlichen Dienst verändern: weg von separierten Gleichstellungsplänen sowie quotierten Einzelfallentscheidungen, hin zu einer systematischen Einbeziehung in längerfristige, allgemeine Personalentwicklungskonzepte. ${ }^{14}$ Dabei würden die bisherigen Entscheidungsquoten künftig als Steuerungsinstrument an Bedeutung verlieren und Gleichstellungspläne eher zu Konzepten werden, wie man Gleichstellungspolitik über gender mainstreaming in alle Ebenen und Formen von Personalentwicklungspolitik integrieren und ein gleichstellungspolitisches Controlling entwickeln könnte.

Insofern ist faktisch auch die Gleichstellungspolitik im öffentlichen Dienst derzeit in einem Umstellungsprozess begriffen, um ein breiteres, der konkreten Situation angepassteres und in das allgemeine Verwaltungshandeln besser integriertes Instrumentarium gleichstellungspolitisch zu erschließen. Druckmittel bleibt allerdings dank der fortbestehenden und insofern mit "harten « inhaltlichen Vorgaben arbeitenden Gleichstellungsgesetze immer die Zielvorgabe des separaten Gleichstellungsplanes, die Kritik und Kontrolle durch Gleichstellungsbeauftragte und der »Rückfall « in den bürokratischen Aufwand einer quotierten Stellenvergabe in jedem Einzelfall. Insofern kann sich Gleichstellungspolitik im öffentlichen Dienst diesen Richtungswechsel zu mehr gleichstellungspolitischer Kooperation und integrierten Lösungen relativ risikolos leisten.

II Vgl. Universitat Hamburg, Arbentsstelle Frauenforderung (Hg.): Frauenforderung an der Universitat Hamburg, 7. Bericht, 1997, Bertcht des Präsidenten S. 6 und Bericht der Frauenbeauftragten S. 4, I 3; Ministerium fur Frauen, Jugend, Wohnungs- und Stadtebau des Landes Schleswig-Holstein (Hg.): Gleichstellung der Frauen im offentlichen Dienst. Erster Gleichstellungsbericht, Kiel I999, S. 40 ff.; Senatsamt fur die Gleichstellung, Hamburg (Hg.): Erfahırungsberıcht uber die Umsetzung des Gesetzes zur Gleichstellung von Frauen und Mànern im hamburgischen offentlichen Dienst (Gleıchstellungsgesetz), Hamburg 1994, S. 5, 22; NN: In Hamburgs Behorden erobern Frauen die Fuhrungsetagen. Aber in gut bezahlten Jobs sind sie in der Minderhert, in: Die Welt v. 3.8.1999; Bundesministerium fur Familie, Frauen und Jugend ( $\mathrm{Hg}$.): Frauen in der Bundesrepublik Deutschland, Bonn 1998, S. 80 f.

I 2 u den seltenen Fallen gehoren ArbG Dortmund, Urt. v. 1. 2. 1992, Az. I Ca 2882/94 sowie VerwG Oldenburg, Beschl. v. 18. 10. 1994, STREIT 1/95 S. 29 f.;

I 3 Die Auseınandersetzung um die prinzipıelle Rechtmaßigkeit von Entscheidungsquoten hat mit EuGH, Urt. v. I 5. 5. 1997 (Marschall), NJW 1997 S. 3429 f. vorerst einen fur Frauen positiven Abschluss gefunden. Seither hat die Auseinandersetzung um die Frage, ob das Dienstalter zugunsten emes Mannes gegen die Quote angefuhrt werden darf, vor den Verwaltungsgerichten jedoch an Dynamı zugunsten der Manner gewonnen. Die gerichtliche Auseinandersetzung um die Quote geht also im Detall weiter.

I4 Vgl. Ministerium fur Frauen, Jugend, Wohnungs- und Städtebau des Landes Schleswig-Holstein a.a.O. (Fn. 9), S. 42; ebenso auch Senatsamt fur die Gleichstellung, Hamburg a.a.O. (Fn.9), S. 2. In dieselbe Richtung allgemeiner auch Schon (Fn. 10), S. $216 \mathrm{ff} ., 313 \mathrm{ff}$. 
Gleichstellungspolitik wäre nicht der erste Bereich, in welchem »verhandeltes Recht«, geschaffen über prozedurales Recht, erprobt würde. Insbesondere auf europäischer Ebene wurde mit der Einführung des Sozialen Dialoges ${ }^{\text {Is }}$ im Bereich der Arbeits- und Sozialpolitik der EU eine Grundlage für die Initiativen neuer Akteure zur Entwicklung normativer Vorgaben gelegt. Man hofft auf diesem Weg, die im Vergleich zur EU-Wirtschaftspolitik vorhandenen Integrationsrückstände im Arbeits- und Sozialbereich schneller aufholen zu können als durch weitere inhaltliche Vorgaben des europäischen Normgebers selbst. Denn im Rahmen des sozialen Dialoges kann den Unterschieden zwischen den Gesellschafts- und Rechtsordnungen der einzelnen EU-Mitgliedstaaten durch größere Problemnähe und Flexibilität besser Rechnung getragen werden - wenn er denn funktioniert, was vom Problemfeld und den dortigen Dialogvoraussetzungen und -begleitumständen abhängt. Aus Beschäftigtensicht zumindest stehen sich heute Erfolge z. B. im Arbeits- und Gesundheitsschutz und Misserfolge z. B. im Bereich der befristeten Beschäftigung gegenüber. Thomas Blanke stellt im vorliegenden Heft der KJ am Beispiel der Europäischen Betriebsräte detailliert dar, unter welchen Voraussetzungen prozedurales Recht, das auf Verhandlungsvorgaben, aber nicht auf Inhalte setzt, Erfolg haben kann - sogar schon, bevor es überhaupt in Kraft getreten ist. Vor allem müsse unter der Drohung »verhandelt, sonst machen wir Gesetze« operiert werden können und dieser »Schatten des Gesetzes« auch greifbar genug sein, um Verhandlungen effektiv zu stimulieren. ${ }^{16}$

Differenziert man die Erfolgsvoraussetzungen für das Schaffen von »verhandeltem Recht" genauer, stößt man auf drei Faktoren:

- Erfolgreiche Verhandlungen sind nur denkbar, wenn zumindest eine der beteiligten Parteien an einem eventuellen Verhandlungserfolg inhaltlich stark interessiert ist und deshalb die Verhandlungsvorgaben auch effektiv nutzt, insbesondere formal erforderliche Anträge stellt, Fristversäumnisse rügt und inhaltliche Vorschläge zum Verhandlungsergebnis macht. Am besten funktioniert prozedurales Recht selbstverständlich, wenn sogar beide Seiten zumindest daran interessiert sind, dass es im Ergebnis überhaupt zu einer einvernehmlichen Regelung kommt.

- Verhandlungen werden nur erfolgreich sein, wenn diese interessierte Verhandlungsseite auch über eine ausreichende soziale Verhandlungsmacht verfügt. Wer keine Macht hat, kann Verhandlungen entweder gar nicht erzwingen oder muss sich deren Ergebnisse faktisch diktieren lassen.

- Fehlende eigene soziale Macht der Verhandlungsakteure kann zum Teil ausgeglichen werden durch Außendruck, insbesondere seitens des Gesetzgebers. Insofern muss der Gesetzgeber selber erkennen lassen, wo und in welche Richtung er Verhandlungen stützen und vorantreiben sowie notfalls inhaltlich im Falle ihres Scheiterns sogar ersetzen will. Hier liegt der eigentliche »Schatten des Gesetzes«, den der gesetzliche Verhandlungsrahmen unabdingbar aufweisen muss, um Erfolgsaussichten zu haben.

Im Fall der Europäischen Betriebsräte gab es mit den nationalen Gewerkschaften und den nationalen betrieblichen Arbeitnehmerinteressenvertretungen hinreichend interessierte Akteure, aus deren Sicht der nationalen Begrenztheit bestehender Mitbestimmungs- und Kontrollregularien dringend abgeholfen werden musste. Diese

Is Art. 139 des Vertrages zur Grundung der Europasschen Gemeinschaft in der Fassung des Vertrages von Amsterdam vom 2. 10. 1997.

16 Vgl. Blanke, Thomas: Recht und Praxıs der Europaischen Betriebsrate, in: KJ ${ }_{4} / 1999$ S. 497 ff. 
Akteure waren auch sozial mächtig in einem weiteren Sinne, selbst wenn der Abschluss einer Vereinbarung zur Einführung Europäischer Betriebsräte im Regelfall wohl kaum durch Streik hätte erzwungen werden können. Beide Verhandlungsseiten stehen allgemein jedoch in vielfältigen, längerfristigen Kooperations- und Konfliktbeziehungen, wo sich die eine Seite immer wieder auch mit der anderen Seite arrangieren muss. Schließlich schuf die Richtlinie zur Einführung Europäischer Betriebsräte einen zeitlich dreifach abgestuften, wohl dosierten Verhandlungsdruck und gab inhaltlich vor, wie Europäische Betriebsräte einzuführen wären, wenn jede Verhandlung scheitern würde. Je schneller die Beteiligten in Verhandlungen eintraten, um so mehr konnten sie den Ablauf der Verhandlungen selber bestimmen, um so eher sie zu Verhandlungsergebnissen kamen, um so eigenständiger und situationsangepasster konnten die konkreten Lösungen vereinbart werden.

Vom Ansatz her ist das Vorhaben einer Einführung Europäischer Betriebsräte durchaus mit dem Anliegen vergleichbar, Gleichstellungspolitik zugunsten von Frauen in privaten Unternehmen über Verhandlungsvorgaben zu implantieren. Allerdings bewegt sich die Implantation Europäischer Betriebsräte auf einem bereits seit einhundert Jahren im nationalen Bereich umkämpften Rechtsgebiet, wobei es mit internationalen Unternehmensausschüssen zumindest von gewerkschaftlicher Seite sektoral auch auf übernationaler Ebene schon über viele Jahre hinweg gute Vorarbeiten gibt. Für Gleichstellungspolitik in der Privatwirtschaft kann Vergleichbares nicht behauptet werden. Sie hätte deshalb tendenziell schlechtere Startvoraussetzungen für Verhandlungen. Erste Gleichstellungsrichtlinien gab es selbst im öffentlichen Dienst erst zu Beginn der achtziger Jahre. Zudem ist Heide Pfarr zuzustimmen, dass die Instrumente und Erfahrungen aus dem öffentlichen Dienst nicht umstandslos auf die Privatwirtschaft übertragen werden könnten. Das gilt um so mehr, als auch im öffentlichen Dienst »im Schatten des Gesetzes « derzeit gerade ein gleichstellungspolitischer Paradigmenwechsel erkennbar wird, wie zuvor dargestellt. Andererseits warb die liberal-konservative Bundesregierung, durchaus keine Vorreiterin yon Gleichstellungspolitik, in der Privatwirtschaft auf der Basis vorausgegangener Modellprojekte und Forschungsaufträge bereits 1985 in den Betrieben mit einem Leitfaden zur Frauenförderung. ${ }^{17}$ Auf über fünfzehn Jahre kann also auch diese Diskussion in Deutschland schon zurückblicken.

Die Frage bleibt, ob die von Pfarr vorgeschlagenen Vorgaben und Strukturen auch einen dem Fall der Europäischen Betriebsräte vergleichbaren ausreichenden Verhandlungsdruck herstellen könnten, ob »der Schatten « stark genug wäre, den ein Gleichstellungsgesetz der von ihr vorgeschlagenen Art »werfen « würde. Und hier sind einige Bedenken angebracht.

\section{Die künftigen Akteure verhandelten Gleichstellungsrechts}

Verhandlungspartner wären nach Pfarr als höchste Ebene die Tarifvertragsparteien, also Gewerkschaften, Arbeitgeberverbände und Arbeitgeber, auf niedrigerer Ebene die am Abschluss von Betriebsvereinbarungen Beteiligten, also Betriebsräte und Geschäftsleitungen, oder schlechtesten Falls, ohne betriebliche Arbeitnehmerinteressenvertretung, der Arbeitgeber allein.

Es liegt auf der Hand, dass es im letzten Fall in aller Regel an einem hinreichend

$17 \mathrm{Vgl}$. Bundesminister fur Jugend, Familie und Gesundheit ( $\mathrm{Hg}$.), Leitfaden zur Frauenforderung in Betrieben, Bonn 1985 . 
interessierten Akteur fehlen wird. Denn die Unternehmen hätten von sich aus auch ohne Gleichstellungsgesetz schon seit Jahren aktiv werden können. Trotz mehr als fünfzehn Jahren öffentlicher Werbekampagnen, Leitfäden zur Frauenförderung, staatlich finanzierter Modellversuche und zuletzt auch Prämierungen z. B. als "frauenfreundlichster Betrieb« bzw. Zertifizierungen mit TOTAL E-QUALITY-Prädikat ist bisher nur eine kleine Minderheit unter den Unternehmen von sich aus in Sachen Gleichstellung der Frau aktiv geworden. Das vielfach vorgebrachte Argument, es läge doch im wohlverstandenen Eigeninteresse der Unternehmen, das auf dem Arbeitsmarkt bzw. im Betrieb vorhandene Arbeitskräftepotential auch unter den Frauen voll auszuschöpfen, ja Frauen seien häufiger sogar die bessere Wahl, zieht ganz offensichtlich in der Praxis bei der großen Mehrheit der Unternehmen nicht.

Bei den wenigen freiwillig Aktiven handelt es sich in der Regel um Großunternehmen. ${ }^{8}$ Von einigen Ausnahmeunternehmen abgesehen ${ }^{19}$ konzentriert sich der große Rest auf vor allem zwei Ansätze: $98 \%$ der frauenfreundlichen Betriebe bieten flexible Arbeitszeiten; wobei allerdings noch genauer zu fragen wäre, ob Frauen und Männer ihre Arbeitszeit auch tatsächlich optional im Interesse ihrer Familie wählen können, was bei den meisten Flexibilisierungsmodellen tatsächlich nicht der Fall ist. ${ }^{20} 80 \%$ bieten im Vergleich zum BErzGG verbesserte Beurlaubungs- oder Rückkehrmöglichkeiten im Zusammenhang mit Erziehungsurlaub. ${ }^{21}$ Eine derartige Beurlaubung von Frauen (Männer beteiligen sich faktisch kaum) wirkt aber zugleich als Freistellung der Betriebe von kollidierenden zeitlichen Anforderungen aus dem familiären Bereich. Durch Beurlaubung bzw, auch Teilzeitarbeit der familiär belasteten Frauen können Betriebe der Notwendigkeit ausweichen, ihre Arbeitsstrukturen selbst familienfreundlicher zu gestalten; insofern also ein gleichstellungspolitisch zwiespältig zu bewertender Ansatz. ${ }^{22}$ Auch bisher bereits positiv in Erscheinung getretene Unternehmen müssten also in den meisten Fällen veranlasst werden, den Rahmen ihrer bisheriger Aktivitäten künftig deutlich zu überschreiten. Hingegen ist Pfarr zuzustimmen, dass das gesetzgeberische Ziel nicht eine Angleichung und Vereinheitlichung bisheriger Praxis sein sollte.

Auch Gewerkschaften und Betriebsräte sind, ungeachtet ihrer gleichstellungspolitischen Verdienste in vielen Einzelfällen, prinzipiell für die innerbetriebliche Durchsetzung von Gleichstellungspolitik problematische Akteure. Gleichstellungspolitik ist nämlich in Zeiten des tendenziellen Stellenabbaus in vielen Bereichen ein Nullsummenspiel: Was den Frauen an Chancen neu gegeben wird, geht den konkurrierenden Männern verloren. Für derartige Umverteilungskonflikte sind Organisationen und Gremien, die vom programmatischen Ansatz her zur Vertretung sowohl von Männer- als auch von Fraueninteressen verpflichtet sind und die nach Mitgliedschaft bzw. Belegschaftszusammensetzung, aber auch ihrer Organisationsgeschichte allzu leicht männerdominiert und männerorientiert agieren ${ }^{23}$, schlecht gerüstet. An

I 8 Vgl. Nather, Brigitte: Frauenforderung in privaten Wirtschaftsunternehmen. Ene Bestandsaufnahme, Universitat Hannover 1991; Bischoff, Sonja: Top-Arbeitgeber für Frauen, Mannheim I996.

19 Vgl. z. B. umfassend Deutsche Telekom (Hg.): Frauenforderkonzept, 2. Aufl. Bonn 1996.

$20 \mathrm{Vg}$ l. z. B. Grobbel, Ute: Flexibilisierung und indıvıduelle Zeitsouveranität, in: Hennıng, Doris/Raasch, Sibylle/Wuttke, Christune (Hg.): Zeitbruche, Hamburg 1998 S. 28 ff.; allgemein: Hamm, Ingo: Flexible Arbeitszeiten in der Prax1s, Frankfurt a.M. 1999, S. 57.

21 Vgl. Bischoff, Sonja: Manner und Frauen in Fuhrungspositionen der Wirtschaft in Deutschland, Köln 1999, S. 58 .

22 In ubrigen lautet ein Ergebnıs der neuesten Befragung von Sonja Bischoff, dass zumındest die bereits in Fuhrungspositionen tatigen Frauen nicht die fehlende Vereinbarkeit von Beruf und Famılie, sondern Vorurteile gegenuber Frauen als großßtes berufliches Hındernis benennen, auch wenn sie selbst Familie haben. Vgl. dies. (Fn. 21 ), S. 47 ff., 54 ff., 58.

23 Die kritische Betrachtung von Pın|, Claudia: Das Arbeitnehmerpatriarchat, Koln 1977 ist inzwischen sicherlich abzuschwachen, aber nicht grundsatzlıch als uberholt zu bezeichnen. Zu.den hessischen 
vielen Formen bestehender Frauendiskriminierung waren und sind Gewerkschaften und Betriebsräte als Vertragspartner von Tarifverträgen oder Betriebsvereinbarungen sogar direkt beteiligt, wie auch Heide Pfarr in anderem Zusammenhang einräumt ${ }^{24}$. Deshalb wird man sich, hier ist dem Fazit von Heide Pfarr (zusammen mit Eva Kocher) durchaus zuzustimmen, im Bereich der Diskriminierung wegen des Geschlechts "nicht allein auf den kollektiven Schutz durch Gewerkschaften und Betriebsräte verlassen können «"s.

Pfarr hofft das Problem dadurch zu lösen, dass die Betriebsräte künftig zwingend nach Geschlechtern quotiert besetzt werden müssen. Als Soll-Vorschrift verlangt $\$$ is Abs. 2 BetrVG eine solche Quotierung entsprechend dem jeweiligen Frauenanteil an der Belegschaft allerdings schon heute, ohne großen tatsächlichen Effekt. Das ist nicht besonders verwunderlich. Denn diese Quotierung belastet die Frauen neben ihrer bisherigen Diskriminierung und der zumeist bestehenden Doppelbelastung durch Beruf und Familie mit weiterer Arbeit, die zudem nicht nur im Interesse der Frauen, sondern im Interesse der Gesamtbelegschaft, also auch der Männer, zu leisten wäre. Wegen dieser Orientierung an der Gesamtbelegschaft fällt es erfahrungsgemäß sogar frauenpolitisch bewusst agierenden Frauen in Betriebsräten schwer, vor sich und anderen im Betriebsrat sog. Fraueninteressen zu vertreten. Denn Fraueninteressen werden aus der Sicht der Betriebsräte strukturbedingt allzu leicht als bloß partikular eingestuft. Insofern wird die primäre Zuständigkeit der Betriebsräte im Bereich der Gleichstellungspolitik aus Sicht der Arbeitgeber wie ein Filter wirken. Zu letzteren dringt nur noch, was die Betriebsräte für sich akzeptiert haben; eine für Frauen missliche Konstellation - der es übrigens zu danken ist, dass in Sachen Gleichstellungspolitik auch Fälle bekannt geworden sind, wo die Geschäftsleitungen weiter zu gehen bereit waren als die Betriebsräte.

Für Gewerkschaften sieht das Eckpunktepapier keine Quoten vor. Allerdings haben sich viele Gewerkschaften inzwischen bereits freiwillig zu einer Quotierung entsprechend ihrem Frauenanteil an der Mitgliedschaft verpflichtet. ${ }^{26} \mathrm{Zu}$ gleichstellungspolitischen Pionieren sind sie deshalb jedoch noch längst nicht geworden, auch dort wo die Quote schon längere Zeit gilt und, seltener, umgesetzt worden ist. Unter Männerdominanz über mehr als hundert Jahre herausgebildete Handlungsformen und -orientierungen lassen sich durch Quotierung zwar leichter in Frage stellen, es bedarf aber auch hier noch vieler Zeit und Argumentationsarbeit sowie struktureller Gegensteuerung über gewerkschaftsinterne Gleichstellungspolitik und eine eigenständige Interessenvertretung für Frauen.

Da allerdings Frauen, allein auf sich gestellt, auch keine innerbetriebliche Verhandlungsmacht entfalten können, wird andererseits trotz aller Vorbehalte und Probleme auf die Unterstützung von Gewerkschaften und Betriebsräten gar nicht verzichtet werden können. Es gibt keine Alternative. Als Sachwalterinnen eines innerbetrieblichen Fraueninteresses ${ }^{27}$ neben Gewerkschaften und Betriebsräten sind jedoch ge-

Personalraten siehe Bednarz-Braun, Iris/Bruhns: Kommunale Frauenpolıtik, München 1995, S. 165 ff. sowie dies. (Fn. 10), S. 204 f., $206 \mathrm{ff}$.

24 Vgl. Pfarr, Heide/Kocher, Eva: Kollektivverfahren im Arbeitsrecht, Baden-Baden I 998 , S. 32 m.w.N.

25 Dies. (Fn. 24).

26 Auf ihrem Gewerkschaftstag im Okt. 1999 hat nunmehr auch die IG Metall ene solche Frauenquote im zweiten Anlauf beschlossen. Bei der kunfrigen Dienstleistungsgewerksclaft verdi zeichnet sich eine Frauenquore entsprechend der jeweiligen Mitgliedschaftszusammensetzung ebenfalls ab.

27 Angesichts der Pluralısierung und Individualisierung von Lebenslagen wird es kunftig zwar schwieriger werden, einheitliche $\gg$ Frauenunteressen $"$ auszumachen. Denn die Unterschede zwischen Frauen sind schon heute beachtlich und nehmen standig zu. Dennoch wird man deshalb nicht sagen konnen, $»$ Fraueninteressen« ließen sıch gar nicht mehr bestimmen oder es gabe keine Unterschiede und Differenzen zu den Männern als Geschlechtsgruppe mehr. Was in einer konkreten Situation ein gleichstellungspolitisches "Frauenınteresse" sein konnte, wird allerdings von Fraueninteressenvertreterinnen nicht mehr einfach 
wählte Gleichstellungsbeauftragte besonders in der Phase der Implementation von Gleichstellungspolitik unverzichtbar. Am Ende mag in Übereinstimmung mit der Fraueninteressenvertretung ein Gleichstellungskonzept entwickelt werden, welches als benannte Frauenbeauftragte eine unternehmensseitige Gleichstellungsmanagerin vorsieht. Am Anfang der Verhandlungen aber müssen die betrieblichen Frauen selbst als gewählte Gleichstellungsbeauftragte oder Gleichstellungsausschüsse Stimme und Verhandlungsrechte bekommen, wenn das vorgeschlagene Verhandlungsmodell überhaupt funktionieren soll.

\section{Machtpotentiale "im Schatten des Gesetzes"}

Zum Aushandeln gleichstellungspolitischer Konzepte haben die von Pfarr vorgeschlagenen Akteure, Gewerkschaften und Betriebsräte, aus eigener Kraft kaum Druckmittel. Den Gewerkschaften stünde zur Durchsetzung eines Gleichstellungstarifvertrages zwar das Streikrecht zur Verfügung. Dies bleibt aber lediglich eine theoretische Möglichkeit. Praktisch ist es derzeit kaum vorstellbar, dass Gewerkschaften finanzielle Ressourcen, vor allem aber Mitglieder in dem für einen Streik erforderlichen Umfang zum Abschluss von Gleichstellungstarifverträgen aktivieren könnten, selbst wenn sie wollten. Realistischer, aber ebenfalls kaum ernsthaft erwartbar wäre höchstens eine Verkoppelung von Gleichstellungspolitik mit sowieso anstehenden Tarifverhandlungen, ähnlich wie derzeit von der IG Metall zur Einführung der Rente mit 60 angedacht. Insofern ist es hoch zu bewerten, dass zumindest den Gewerkschaften NGG und HBV bereits der Abschluss einiger Rahmenvereinbarungen zur Frauenförderung gelang. ${ }^{28}$

Betriebsräte haben bereits nach dem geltenden BetrVG einige eigene Einwirkungsmöglichkeiten ${ }^{29}$ auf die Gleichstellungspolitik von Unternehmen. Pfarr schlägt vor, diese über Initiativrechte im Bereich der zwingenden Mitbestimmung mit Verfahren bis hin zur Einigungsstelle auszubauen. Schon die Tatsache, dass die meisten heute existierenden verbindlichen Regelungen zur betrieblichen Frauenförderung in Form von Betriebsvereinbarungen abgeschlossen worden sind ${ }^{30}$ deutet darauf hin, dass die wichtigeren Akteure verhandelter Gleichstellung künftig tatsächlich nicht die Gewerkschaften, sondern die Betriebsräte sein werden. Deren Rechte auszubauen ist deshalb ein vernünftiger Weg, was die Aufnahme von Verhandlungen betrifft.

Um Inhalte, insbesondere etwas gravierendere betriebliche Strukturveränderungen im Sinne von gleichstellungspolitischen Innovationen, durchzusetzen, ist der Weg über Betriebsräte hin zur Einigungsstelle jedoch weniger aussichtsreich, solange der Gesetzgeber zu diesen Inhalten weiter schweigt und die Interessenvertretung der Frauen bei den Verhandlungen selber durch konkrete Rechte keine Stimme erhält. Es steht wegen ihrer internen Zerrissenheit zwischen Männer- und Fraueninteressen kaum zu erwarten, dass die Betriebsräte von sich aus besonders weitreichende gleich-

von oben entschieden und dann reprasent1ert werden konnen. Es ware zumeist erst im Diskurs mit den betroffenen Frauen zu ermitteln. Gleichstellungsbeauftragte sind dann jedoch nach dem oben Ausgefuhrten die geetgneteren Initıatorinnen und Moderatorinnen fur diesen Frauendiskurs.

28 Vgl. Zauner, Margrit: Forderung von Managerınnen. Frauenforderplane als Mittel zur Erschließung weiblicher Fuhrungskrafteressourcen, Munchen und Mering I990, S. 87; Nather (Fn. I 8), S. Io2 ff.

$29 \mathrm{Vgl}$. z. B. schon Bertelsmann, Klaus: Enflussmoglichkeiten von Betriebsraten zur Forderung der Gleıchstellung von Frauen und Mannern im Arbeitsleben, Hamburg 1981 (!), Informationspapier, hg. von der Leitstelle Gleichstellung der Frau, Hamburg; Fisahn, Andreas: Rechtliche Bedingungen von Frauenforderung im Betrieb, in: WSI Mitt 1/1995 S. $22 \mathrm{ff}$.

30 Vgl. Nather (Fn. 18); Zauner (Fn. 28). 
stellungspolitische Vorschläge machen werden. Und die betriebsverfassungsrechtliche Einigungsstelle hat sich bisher vor allem dort als Durchsetzungs- und Gestaltungskraft erwiesen, wo es um die Abwehr unternehmerischer Einzelfallentscheidungen, völliges unternehmerisches Scheitern/Sozialpläne sowie um soziale Angelegenheiten im Sinne von Verteilungsstreitigkeiten ging. Personalplanung, um die es bei innerbetrieblicher Gleichstellungspolitik vorrangig gehen dürfte, Berufsbildung und wirtschaftliche Entscheidungsfragen, also die zukunftsweisende Unternehmenspolitik, gehören bisher betriebsverfassungsrechtlich nicht in den Zuständigkeitsbereich von Einigungsstellen. Hier dürfte die richterlich-schlichtende Sichtweise einer Einigungsstelle nicht unbedingt ein Garant für gute Ergebnisse und insbesondere Kreativität sein. Über das, was Betriebsräte bzw. Geschäftsleitung von sich aus vorgeschlagen haben, könnte eine Einigungsstelle sowieso nie hinausgehen.

Im übrigen erfasst das betriebsverfassungsrechtliche Instrumentarium nicht die Fälle, wo es auf Grund der Betriebsgröße oder aber einer schwachen bzw. uninteressierten Belegschaft gar keinen Betriebsrat gibt. Frauen sind jedoch viel häufiger als Männer gerade in solchen Unternehmen tätig. Hier ist also besonders zu befürchten, dass die Unternehmen gar nicht oder mit bequemen, aber wirkungslosen Mitteln tätig werden.

Dennoch will Pfarr die Gleichstellungsgesetzgebung inhaltlich auf ein Minimalprogramm (Nr. 10, 1 r) beschränken: Pflicht zur Analyse der Beschäftigungssituation, Gleichstellungspläne (Inhalt offen), Anhörungs-, Initiativ- und Schutzrechte für eine Fraueninteressenvertretung (die aber entgegen Nr. 11 niemals allein aus einer benannten Frau bestehen dürfte). Ein darüber doch hinausgehender gesetzlicher Effektivitätsschutz durch inhaltliche Vorgaben wird später zwar angedacht (Nr. 20, $2 \mathrm{r}$ ), bleibt bisher aber völlig vage und dürfte so nicht greifen.

\section{I Verbandsklage}

Als eigentlichem Druckmittel setzt Pfarr auf die Verbandsklage, erhoben durch Gewerkschaften oder autorisierte Frauenverbände (Nr. I7) sowie auf Sammelklagen diskriminierter Frauen (Nr. 18). Dabei sollte in die Betrachtung des Eckpunktepapiers dasjenige mit einbezogen werden, was Heide Pfarr und Eva Kocher anderen Orts $^{3 '}$ bereits ausführlicher zu diesen Klagemöglichkeiten entwickelt haben. Da die Autorinnen gerade im Fall von Frauendiskriminierung Gewerkschaften auch als Klägerinnen wegen der in ihnen repräsentierten widerstreitenden Interessen nicht in vorderster Front sehen ${ }^{32}$, wäre hier vor allem eine Einbeziehung von Frauenverbänden wichtig. Tatsächlich sehen die Autorinnen neben dem Deutschen Juristinnenbund (DJB) allerdings derzeit keine weiteren, entsprechend leistungsfähigen Frauenverbände. ${ }^{33}$

Es könnten sich jedoch angesichts neuer Aktionsmöglichkeiten auch neue Verbände entwickeln. Die Frauenbewegung hat z. B. im Frauenhausbereich bewiesen, dass sie sich problembezogen sehr wohl fachkompetent und durchsetzungsstark organisieren kann. Gerade in der Rechtsberatung könnte an die erfolgreiche Tradition früherer autonomer Rechtsberatungsstellen von und für Frauen angeknüpft werden. ${ }^{34}$ Insofern könnte die Einführung von Verbands- und Gruppenklage einen wichtigen Schritt

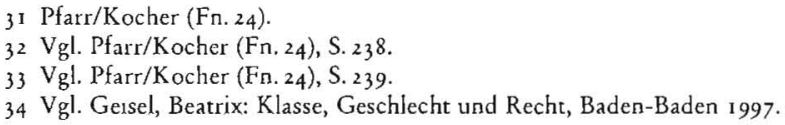


bilden, um Gleichstellungspolitik in der Privatwirtschaft mehr Nachdruck zu verleihen.

Ein Blick auf die Frauenrechtsschutzbewegung des letzten Jahrhunderts und in die USA zeigt jedoch, dass effektive Strukturen über Frauenverbände und -gruppen nicht schnell aus dem Boden zu stampfen sind, sondern erst in einem längeren Prozess entwickelt werden müssten. Deshalb wäre eine Verstärkung durch die Möglichkeit zur Behördenklage wichtig. Pfarr/Kocher halten die derzeit bestehende Fraueninfrastruktur im öffentlichen Dienst hierfür allerdings wegen ihrer Heterogenität für ungeeignet. ${ }^{35}$ Zumindest kommunale Gleichstellungsstellen und die Einrichtungen auf Landesebene in den Frauenministerien ließen sich jedoch in diese Richtung ausbauen. Das Problematischste an einer Einführung der Behördenklage dürfte gegenwärtig natürlich sein, dass die Einführung gerichtlich schlagkräftiger Gleichstellungsbehörden Geld kostet und deshalb mit der aktuellen Sparpolitik im öffentlichen Dienst kollidiert.

Die von Pfarr vorgeschlagene Beweislastumkehr für Diskriminierungsklagen nach \$ I I a BGB gegen Unternehmen, die Gleichstellungspläne nicht haben oder nicht einhalten, wäre in diesem Zusammenhang ebenfalls hilfreich. Denn dadurch könnten die Erfolgsaussichten von Diskriminierungsklagen deutlich verbessert werden. Hinzukommen müssten jedoch höhere Schadensersatzdrohungen sowie die Möglichkeit, über Vergleiche nicht nur Geld, sondern auch Gleichstellungsmaßnahmen vereinbaren zu können, wie das Pfarr/Kocher, orientiert an amerikanischen Erfahrungen, ausführlicher entwickelt haben. ${ }^{36}$

Nach den Eckpunkten allerdings wäre derzeit nicht mit einem sehr durchsetzungsstarken Mittel zu rechnen. Die Einführung der Verbandsklage könnte die Anwendungsschwäche eines rein verfahrensmäßig ausgerichteten Gleichstellungsrechts nur sehr unzulänglich ausgleichen. Pfarr konstatiert schon selber, dass diese Klagen sämtlich nur in dem Fall Druck erzeugen könnten, wo ein Unternehmen gar keinen Gleichstellungsplan entwickelt oder einen bestehenden - nachprüfbar - nicht eingehalten hat. Damit ist jedoch die gleichstellungspolitische Lücke bei der inhaltlichen Qualitätskontrolle von Gleichstellungsplänen gar nicht gefüllt. Im klein- und mittelbetrieblichen Bereich würden Sammel- und Verbandsklagen ebenso wie die Mitbestimmung der Betriebsräte nur schlecht greifen. Denn auch dieses Instrument ist eher auf die Kontrolle von Großbetrieben hin ausgelegt. Dort kann sich der zur Klage notwendige kollektive Bezug einer Diskriminierung leichter herstellen lassen, und die klagebefugten Verbände werden auch eher von einer Diskriminierung erfahren. Schließlich kann eine Diskriminierungsklage trotz Beweislastumkehr immer nur da greifen, wo tatsächlich diskriminiert wird. Ein insoweit in der Personalpolitik fehlerfrei arbeitendes Unternehmen ist also auf dem Klageweg in seiner gleichstellungspolitischen Inaktivität nicht zu beeinflussen.

\subsection{Subventionen und öffentliche Aufträge}

Um dem neuen Gleichstellungsansatz Nachdruck zu verleihen, setzt Pfarr daneben noch auf die schon vielfach geforderte Verkoppelung von öffentlicher Geldvergabe mit Gleichstellungspolitik (Nr. Ig). Diesem Weg käme deshalb besondere Bedeutung zu, weil er unabhängig von der Existenz von Betriebsräten wirkt und deshalb auch

35 Vgl. Pfarr/Kocher (Fn. 24), S. 240.

$36 \mathrm{Vg}$. Pfarr/Kocher (Fn. 24), S. 244. 
Klein- und Mittelbetriebe etwas besser erfasst würden. Bedauerlicherweise erwähnt das neue Regierungsprogramm »Frau und Beruf « diesen Weg im Zusammenhang mit dem Gleichstellungsgesetz gar nicht mehr und formuliert später nur noch einen lapidaren Prüfauftrag. ${ }^{37}$ Das ist insofern unverständlich, weil nach der EuGH-Rechtsprechung ${ }^{38}$ und verschiedenen Anhörungen auf Länderebene ${ }^{39}$ schon sehr viel geprüft worden ist mit dem Ergebnis, dass zumindest als Auflage das Vorhandensein eines Gleichstellungsplanes durchaus in Vergabeverfahren eingeführt werden könnte. ${ }^{40}$

Auch im Zusammenhang mit der Vergabe öffentlicher Gelder wird jedoch schnell klar, dass der Gesetzgeber nicht umhin kommt, inhaltlich zu regeln, was er unter effektiver Gleichstellungspolitik versteht. Ansonsten könnte ein Unternehmen jeden beliebigen Gleichstellungsplan einreichen, selbst »heiße Luft«, wie auch Pfarr befürchtet. Dementsprechend kommt sogar das Eckpunktepapier ganz am Ende zu der Erkenntnis, dass der Gesetzgeber doch inhaltliche Mindeststandards formulieren müsste, an denen Gerichte in Fall von Diskriminierungs- und Auftragsvergabeklagen messen könnten, ob ein Gleichstellungsplan im Sinne des Gleichstellungsgesetzes vorliegt. Der von Pfarr hierzu vorgeschlagene Beispielskatalog verschiedener Vorgehensweisen und Mittel hilft dabei allerdings kaum weiter. Auch ein solcher ließe nämlich offen, wieviele Beispiele ein Unternehmen in seiner konkreten Situation umzusetzen oder durch Gleichwertiges zu ersetzen hätte. Hier liegt die größte Schwachstelle der Eckpunkte und der Einführung von gleichstellungspolitischer Normierung über Verhandlungen allgemein.

Pfarr hofft auf die Rechtsprechung, welche diejenigen Maßstäbe für Einwirkungsintensität und Regelungsdichte entwickeln solle, zu deren Entwicklung sich der Gesetzgeber offenbar nicht in der Lage sah - ein rechtsstaatlich betrachtet fatales Verfahren, das erneut zur Entparlamentarisierung politischer Entscheidungsfindung beitragen würde. Auch inhaltlich dürften die Hoffnungen nicht hoch angesetzt werden. Die gleichstellungsfreundlichen Entscheidungsimpulse der letzten Jahre kamen zumeist nicht von den nationalen Zivil- und Arbeitsgerichten (und schon gar nicht aus der Verwaltungsgerichtsbarkeit!), sondern vom EuGH. Letzterer aber wäre für die Konkretisierung eines deutschen Gleichstellungsgesetzes unzuständig.

Auch in diesem Zusammenhang seien nochmals Pfarr/Kocher angeführt: »Generell kann man sich für die Wirksamkeit von gleichberechtigungs- und gleichstellungsorientiertem Recht ohnehin nicht allein auf den justizförmigen Weg verlassen. Die Ursachen yon Frauendiskriminierung reichen weit in die gesellschaftlichen Wertvorstellungen und persönlichen Verhaltensweisen hinein. Wirksame Durchsetzungsmechanismen müssen deshalb Raum schaffen für das selbständige und selbstbewusste Handeln von Frauen und ihre Konflikt- und Durchsetzungsfähigkeit fördern. «4' Dem ist nichts hinzuzufügen. Das Eckpunktepapier aber, das die Frauenbeauftragten und -ausschüsse in den Hintergrund drängt und die Durchsetzung effektiver Frauenförderung den Inhalten nach letztendlich ohne gesetzgeberische

37 Vgl. Bundesministerium fur Familie, Senioren, Frauen und Jugend (Fn. 2), S. 22.

38 Vgl. EuGH, Urt. v. 20. 9. 1988, NVwZ 1990, S. 353 ff.

39 Siehe z. B. Mrnisterium fur Frauen, Jugend, Familie und Gesundheit des Landes Nordrhein-Westfalen (Hg.): Mehr Chancengleichheıt im Beruf - Freiwilligkeit versus Auflagen fur eine Frauenforderpolitik, Dusseldorf 1998 .

$40 \mathrm{Vgl}$. insbesondere den Beitrag von Gisela Lange fur die Europaische Kommission, Brussel, auf der Anhorung in Nordrhein-Westfalen (Fn. 39), S. so ff.; Bottger, Karin: Frauenforderung durch öffentliche Auftragsvergabe, in: ArbuR 1996 , S. $x_{4} 8 \mathrm{ff}$.; Degen, Barbara: Staatliche Wirtschaftsforderung und Art. 3 Abs. 2 GG, in: STREIT 1996, S. 3 ff.

4I Pfarr/Kocher (Fn. 24), S. 239. 
Vorgaben an Einigungsstellen und Justiz verweist, wird diesem Anspruch nicht gerecht.

\section{Alternativen}

Damit ist ein besserer Weg zur Gleichstellungspolitik im Bereich der Privatwirtschaft natürlich noch nicht gefunden. Zu suchen wäre er neben den im Vorangegangenen vorgeschlagenen Detailverbesserungen insbesondere in einer effektiveren gesetzlichen Absicherung von Gleichstellungsbeauftragten und Gleichstellungsausschüssen in den Betrieben. Sie müssten schon zu Beginn an den innerbetrieblichen Verhandlungen mit »starken« eigenen Rechten beteiligt sein. Neben bloßen Initiativrechten sollten die Interessenvertretungen der Frauen das Recht erhalten, Betriebsvereinbarungen und einseitig vom Arbeitgeber erlassene Gleichstellungspläne vor den Arbeitsgerichten einer Billigkeitskontrolle unterziehen zu lassen. Diese effektivere Verankerung einer Fraueninteressenvertretung würde Frauen eine interne Moderation zur Ermittlung ihrer Interessen $n^{42}$ bringen sowie eine Stimme bei den Verhandlungen geben, ihr Machtpotential allerdings selbst bei guter verfahrensmäßiger Absicherung noch nicht einmal auf das Niveau der Betriebsräte heben.

Damit der »Schatten des Gesetzes « für das Aushandeln von Gleichstellungsregelungen innerbetrieblich zur Vereinbarung effektiver Inhalte ausreicht, muss der Gesetzgeber deshalb Handlungsdruck in Richtung auf ein bestimmtes Mindestniveau schaffen und dafür auch selbst inhaltlich Farbe bekennen. Sogar im Rahmen von Verbandsklage oder Verkoppelung mit öfentlicher Autragsvergabe stellt sich, wie dargestellt, immer wieder die Frage, was ein effektiver Gleichstellungsplan sein soll und woran Gerichte oder Vergabebehörden das messen könnten. Damit wende ich mich nicht dagegen, dass im Bereich der Privatwirtschaft Gleichstellungspolitik künftig vorrangig ausgehandelt und betriebsangepasst vereinbart werden soll. Wenn die neuen gleichstellungspolitischen Akteure, Tarifvertragsparteien, Betriebspartner, Frauenvertreterinnen und Arbeitgeber Gleichwertiges oder gar Besseres zu bieten haben, würden die inhaltlichen Vorgaben des Gesetzgebers ja wieder zurücktreten.

$\mathrm{Zu}$ denken wäre hier nun doch an eine Übertragung der im öffentlichen Dienst erprobten Ansätze: Diese haben sich zwar als langsam und bürokratisch erwiesen, aber immerhin Erfolge vorzuweisen. Gerade die in Unternehmen und Betriebsräten so unbeliebte Frauenquote ${ }^{43}$, wie sie in den früheren Entwürfen von SPD und Bündnis 9o/DIE GRÜNEN mit Sanktionen versehen vorgeschlagen wurde ${ }^{44}$, wäre ein effektives Druck- und Motivationsmittel, in den Betrieben von sich aus nach besseren Lösungen zu suchen und damit einer Entscheidungsquote unter Qualifikationsvorbehalt auszuweichen.

Benötigt würde ausserdem eine Stelle, die diese betrieblichen Einzellösungen als dem Gesetz gleichwertig prüft. Zu denken wäre dabei an Zertifizierungen, wie sie im TOTAL E-QUALITY-Bereich bereits heute erprobt werden. Ähnlich wie im Fall der Europäischen Betriebsräte wäre an ein zeitlich gestuftes Verfahren zu denken, in welchem ein solches abweichendes Vorgehen ausgehandelt werden könnte. Bis zur Errichtung einer Zertifizierungsstelle könnte z. B. das Einvernehmen zwischen Betriebsrat, Gleichstellungsbeauftragter und Geschäftsleitung für die Unterstellung

42 Vgl. schon Fn. 27

43 Vgl. die sehr differenzierte Konfliktanalyse bei Bednarz-Braun/Bruhns (Fn. Io), S. 162 ff., I 83 ff., 209 f. Siehe auch Schon (Fn. 10), S. 304

44 Vgl. Fn. 5 und 6. 

der Sicht von Gerhard Leibholz

\author{
Zu den Ursprüngen der Parteienstaatslehre
}

Im Jahre 1928 hielt der Staatsrechtler und spätere Bundesverfassungsrichter Gerhard Leibholz seine Antrittsvorlesung, die unter dem Titel "Zu den Problemen des faschistischen Verfassungsrechts" publiziert wurde. Diese Untersuchung stellt einen weitgehend verkannten Schlüssel zum Verständnis von Leibholz' Parteienstaatslehre dar: Es gelingt nämlich anhand der zeitgenössischen deutschsprachigen und italienischen Literatur der Nachweis, daß Leibholz seine Parteienstaatslehre unter anderem am Vorbild des faschistischen Einparteiensystems orientierte. Die Untersuchung ist nicht nur von rechtshistorischem Interesse, sondern weist auch einen aktuellen rechtlichen Bezug auf, da Leibholz Theorie nachhaltig die Rechtsprechung des Bundesverfassungsgerichts und damit nicht zuletzt die heutige Sichtweise der politischen Parteien beeinflußt hat.

Die Studie wendet sich daher sowohl an Staats- und Verfassungsrechtler als auch an Politologen und (Rechts-)Historiker.

1999, 187 S., brosch., 58,-DM, 423,- öS, 52,50 sFr,

ISBN 3-7890-6307-X

(Schriften zum Parteienrecht, Bd. 23) 